\title{
Qu'est-ce qu'une bonne annonce de pharmacovigilance?
}

\author{
EIViS arrive bientôt: en octobre 2014, Swissmedic va mettre en place un système de \\ saisie en ligne (Electronic Vigilance System) d'effets indésirables (EI) suspectés des \\ médicaments. Ci-dessous, nous rappelons quelles annonces sont particulièrement \\ importantes pour la sécurité des médicaments.
}

Rudolf Stollera,

Karoline Mathys ${ }^{b}$

Martina Schäublinc,

Christoph Küngd

a Senior Expert, Division Sécurité des médicaments, Swissmedic

b Dr pharm., Cheffe du Secteur Surveillance du marché, Swissmedic

c Dr méd., Cheffe de l'unité Vigilance, Division Sécurité des médicaments, Swissmedic

d Dr phil., Chef de la Division Sécurité des médicaments, Swissmedic
Correspondance:

Dr Martina Schäublin

Swissmedic

Hallerstrasse 7

Case postale

CH-3000 Berne 9

Tél. 0584638623

martina.schaeublin[at]

swissmedic.ch
A compter d'octobre 2014, le nouveau système ElViS permettra aux professionnels de santé d'annoncer en ligne les effets indésirables de médicaments. ElViS va établir un lien direct entre eux et les centres régionaux de pharmacovigilance (CRPV) et améliorera ainsi l'échange d'informations avec Swissmedic. Des représentants de la FMH, de PharmaSuisse et de la GSASA (Association suisse des pharmaciens de l'administration et des hôpitaux) ainsi que les CRPV ont été et sont impliqués dans le développement du programme et des tests. Médecins et pharmaciens seront également formés à utiliser le système: ElViS est un système en grande partie intuitif et proposera, si nécessaire, une aide à la saisie. Swissmedic rappelle aujourd'hui quelles sont les annonces d'EI particulièrement importantes pour la sécurité des médicaments et les points essentiels lors de la saisie. Une description ciblée des EI est déterminante. Pour une présentation plus complète, veuillez consulter la publication parue dans le BMS 4/2013 [1].

Les annonces spontanées, en Suisse et à l'échelle internationale (notamment dans le cadre du «WHO Programme for International Drug Monitoring»), demeurent un instrument déterminant pour identifier rapidement des signaux de sécurité concernant les médicaments sur le marché et réduire les risques y afférents. Elles reposent sur la collaboration et la compréhension des professionnels de santé qui transmettent «une bonne annonce de bonne qualité au bon moment» au sens des «Bonnes Pratiques de Pharmacovigilance». Il est important de signaler le caractère inhabituel de l'EI ainsi que d'annoncer les EI graves.

\section{Objectif: identifier au plus tôt les signaux de sécurité et prendre des mesures de réduction des risques}

Les annonces spontanées ont pour objectif d'améliorer les connaissances des risques médicamenteux connus et d'identifier de nouveaux effets indésirables jusque-là inconnus.

En conséquence, un signal de sécurité est défini comme: un nouvel aspect suspecté d'un risque connu (le cas normal en pharmacovigilance) ou

- un nouveau risque suspecté, jusque-là inconnu.

Les annonces spontanées à elles seules ne font pratiquement jamais la preuve d'un EI. Un signal qui en résulte est en premier lieu une suspicion qui doit encore être confirmée. Dans cette hypothèse, il faut prendre des mesures de réduction des risques. Plus le potentiel lésionnel est élevé (par exemple, problème difficilement ou tardivement identifiable, lésion grave du patient, grand nombre de personnes exposées), plus les mesures doivent être rapides et incisives.

Citons à titre d'exemple le risque connu et significatif des hémorragies sous anticoagulant oral; la pharmacovigilance en Suisse et à l'échelle internationale doit prendre en compte en permanence les éléments suivants: que les anticoagulants soient nouveaux ou déjà connus, les signaux concernent par exemple les interactions pharmacodynamiques avec d'autres médicaments comme l'héparine, l'aspirine, les anti-inflammatoires non stéroïdiens, les antidépresseurs de type ISRS, ainsi que les interactions pharmacocinétiques (via des enzymes CYP, des transporteurs comme les OATP ou l'influence de la fonction rénale). Des problèmes supplémentaires concernant les nouveaux anticoagulants résultent de l'absence d'antidote reconnu en cas de surdosage ou d'hémorragie aiguë; les annonces actuelles concernent entre autres les problèmes en cas de changement d'anticoagulant et ceux liés au suivi thérapeutique. La seule annonce d'une complication hémorragique ne suffit pas. Il faut également documenter les aspects qui viennent d'être cités. A ce sujet, Swissmedic a publié une analyse des annonces d'EI etinformé sur les précautions à prendre [2]. L'information professionnelle et celle destinée aux patients sont régulièrement contrôlées et mises à jour en conséquence.

A titre d'exemple de la deuxième catégorie (EI nouveaux et inattendus), citons l'apparition d'une narcolepsie à la suite d'une vaccination contre la grippe pandémique H1N1 en 2009 avec Pandemrix ${ }^{\circledast}$, 
Figure 1

L'organisation de la pharmacovigilance en Suisse reste inchangée.

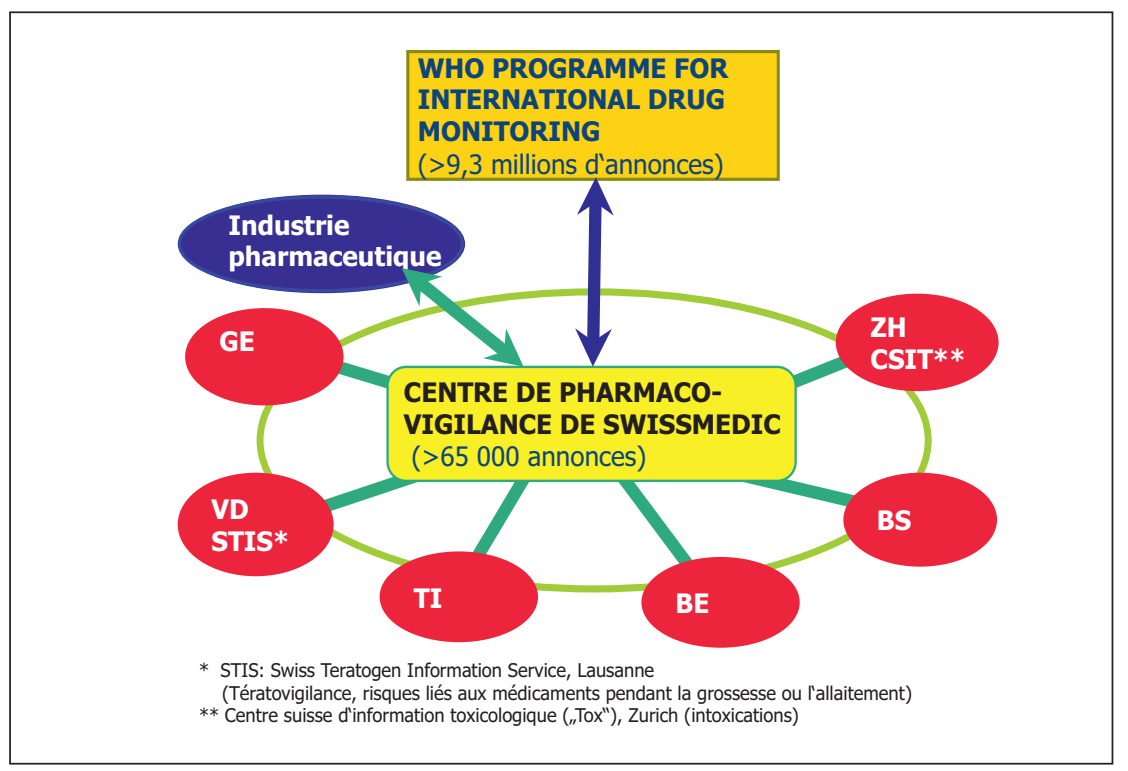

un vaccin pandémique avec adjuvant. Les personnes concernées n'ont développé la maladie que plusieurs mois après la vaccination. Le risque n'a pu être identifié que grâce à une grande attention des annonceurs et l'augmentation du nombre d'annonces, dans un premier temps dans les pays scandinaves. Au total en Suisse, 9 cas faisant suite à l'injection d'un vaccin pandémique ont été rapportés jusqu'à présent. Des études épidémiologiques détaillées révèlent maintenant l'existence d'un lien entre la narcolepsie et le vaccin Pandemrix ${ }^{\circledast}$. Ce dernier n'a été utilisé que pendant la pandémie grippale et n'est plus sur le marché.

\section{La bonne annonce d'EI}

Les caractéristiques des signaux sont les suivantes:

- il s'est produit quelque chose d'inattendu. Soit, il s'agit d'un EI connu, mais comportant de nouveaux aspects, mentionnés plus ou moins clairement dans l'information professionnelle, mais qui posent problème dans la pratique quotidienne (par ex. absence d'antidote, de monitoring thérapeutique). Soit, l'EI est en soi nouveau et inhabituel;

- il s'agit de risques significatifs, potentiellement graves.

Ce sont donc ces situations, aspects ou expériences inhabituels ou inhabituellement graves liés à des EI qui déclenchent une annonce et devraient être particulièrement soulignés dans la description. Le système en ligne transférera le contenu dans la base de données. L'annonce devrait contenir les caractéristiques les plus importantes de l'événement, notamment en ce qui concerne

- l'intensité et la gravité de l'EI (exemple: hépatite avec ictère lorsque l'information professionnelle ne mentionne qu'une élévation des transaminases);
- la chronologie (exemple: atteintes hépatiques jusqu'à 6 semaines après l'arrêt d'un traitement par co-amoxiclav, flucloxacilline ou substances apparentées);

- les facteurs prédisposants (exemple: insuffisance rénale - intoxication au lithium);

- les problèmes liés à l'utilisation (exemple: prise quotidienne au lieu d'une prise hebdomadaire de méthotrexate à faible dose dans une indication rhumatologique ou dermatologique, ayant pour conséquence une intoxication accidentelle grave).

Pour la conduite à tenir voir l'encadré.

Annoncer un El: quoi, quand et comment? L'obligation d'annoncer pour les professionnels de santé est énoncée à l'article 59 de la loi sur les produits thérapeutiques (LPTh).

\section{Quels EI faut-il annoncer, dans quels délais?}

El graves, dans un délai de 15 jours

- fatals ou engageant le pronostic vital

- séquelles durables, hospitalisation / hospitalisation prolongée

- El «médicalement importants» (exemples: traitement d'une crise d'épilepsie ou d'une hypoglycémie au cabinet médical de sorte que des conséquences plus graves sont évitées; échec d'un vaccin)

Nouveaux El, dans un délai de 60 jours s'ils ne sont pas graves

Comment annoncer?

Saisie structurée selon formulaire (à l'avenir de préférence en ligne)

- informations relatives au patient, date, gravité de l'EI

- médicaments suspectés, avec dosage et indication

- autres médicaments

- facteurs prédisposants (par exemple, fonction rénale - créatinine ou clairance de la créatinine avant l'EI)

Description de l'El (texte libre)

- diagnostic et symptômes / résultats significatifs

- aspects inopinés de l'El à souligner

- évolution de l'El en relation avec l'exposition médicamenteuse (temps écoulé jusqu'à l'apparition, jusqu'à l'amélioration après l'arrêt - du ou des médicament(s)

- diagnostic différentiel - causes non médicamenteuses (par exemple, en cas de lésions hépatiques, consommation d'alcool, obstruction des voies biliaires, sérologies virales) - problèmes au niveau de la prescription, de la distribution ou de l'usage 
Figure 2

Des annonces toujours plus fréquentes: nombre d'annonces de pharmacovigilance par an.

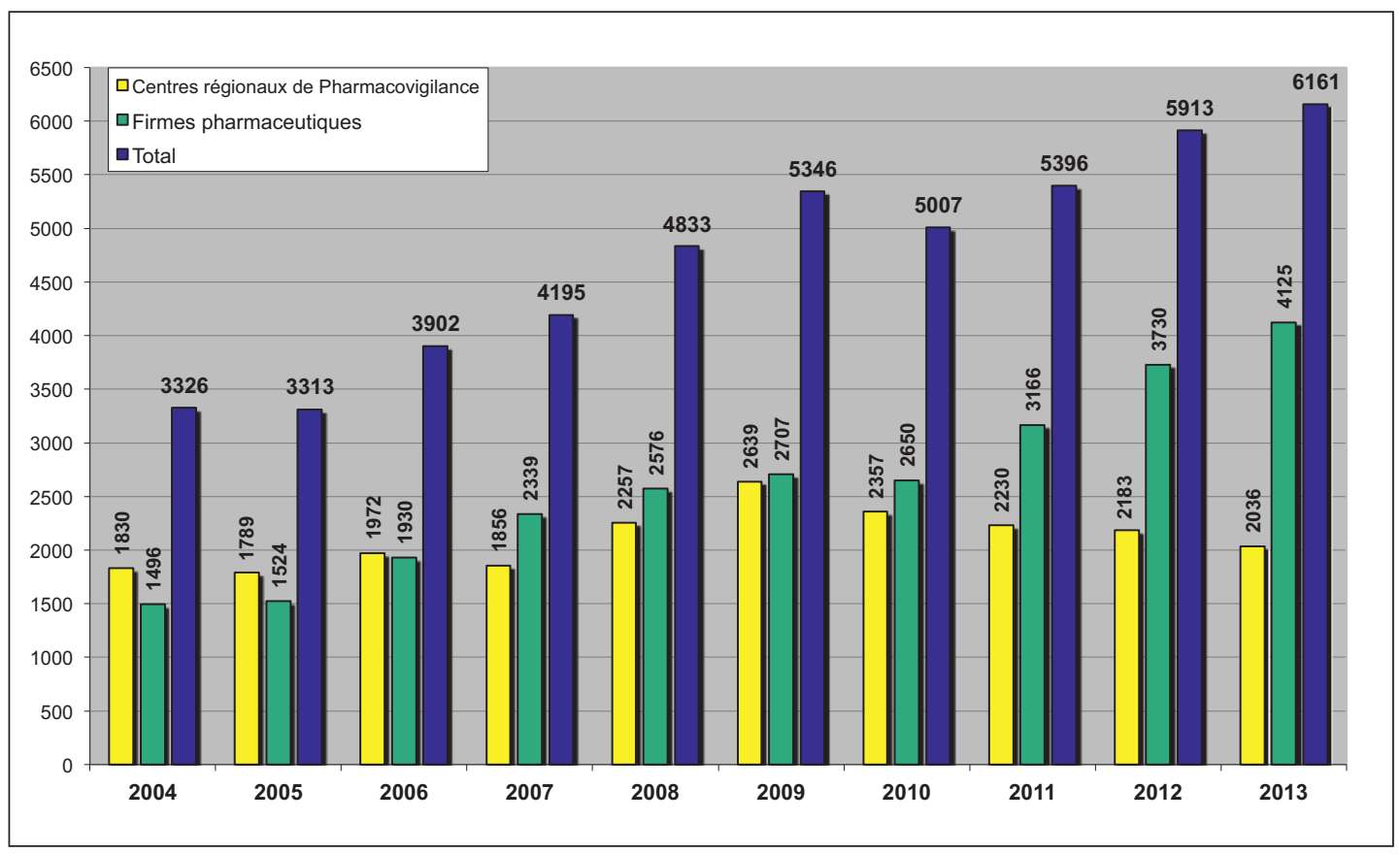

\section{L'organisation de la pharmacovigilance en Suisse reste inchangée (figure 1)}

Les annonces seront toujours adressées aux centres régionaux de pharmacovigilance, même après l'introduction de la saisie en ligne. Elles continueront d'être enregistrées sous forme totalement anonyme pour ce qui est des patients, des annonceurs, des professionnels et institutions impliqués dans la base de données de Swissmedic et dans les bases de données internationales de l'OMS ainsi que d'être transmises aux firmes concernées.

\section{Des annonces toujours plus fréquentes}

Le nombre d'annonces continue de progresser. Selon les statistiques de l'OMS de mars 2014, la Suisse occupe en Europe la deuxième place après le Danemark en ce qui concerne le nombre d'annonces d'El par million d'habitants. Mais leur qualité n'en est pas moins importante. Nous remercions les médecins et pharmaciens annonceurs de contribuer à ce qu'ElViS, le système de transmission électronique des annonces de pharmacovigilance, puisse transmettre à compter du mois d'octobre 2014 de bonnes annonces, de bonne qualité, au bon moment.

\section{Références}

1 Levy G, Caduff P, Stoller R. Pharmacovigilance et annonces spontanées d'effets indésirables: 10 ans après la mise en vigueur de la Loi sur les Produits Thérapeutiques. Bull Méd Suisses. 2013;94(4):101-4.

2 HPC - Swissmedic informe du risque de saignements sévères en relation avec la prise de dabigatran $\left(\right.$ Pradaxa $\left.{ }^{\oplus}\right)$ : mises en garde et recommandations thérapeutiques. 12.4.2013. 